\title{
Ossifying fibroma of the maxilla: a rare case
}

\begin{abstract}
Ossifying fibroma(OF) is primarily classified under Fibro osseous lesions(FOL's) of the jaw: the rare, benign, non aggressive tumors. This article presents a case of ossifying fibroma with minimal symptoms involving the left maxilla in a 66 year old female patient. The clinical and diagnostic features, management and prognosis were discussed along with the review of literature.
\end{abstract}

Keywords: ossifying fibroma, fibro osseous lesion, maxilla

\author{
Volume 3 Issue 2 - 2017 \\ Priyanka Bhat,' Atul Kaushik, ' Vinod VC,' K \\ Sridevi, ${ }^{2}$ Astha Chaudhry' \\ 'Faculty of Dental Sciences, SGT University, India \\ ${ }^{2}$ Lenora institute of Dental sciences, India
}

\begin{abstract}
Correspondence: Astha Chaudhry, Department of Oral Medicine, Diagnosis and Radiology, Faculty of Dental Sciences, SGT University, Farukh Nagar Road, Budhera, Gurgaon, Haryana, India, I2200I, Email dr.asthac@gmail.com
\end{abstract}

Received: October 28, 2016 | Published: June 08, 2017

\section{Introduction}

Ossifying fibroma is benign, uncommon, monostotic well defined usually unilocular but occasionally multilocular fibro-osseous tumor, arising from the cells of periodontal ligament and is composed of fibrous connective tissue with variable amounts of calcified tissue resembling bone, cementum or both. It is generally an asymptomatic, slow growing lesion, found in $2^{\text {nd }}$ to $4^{\text {th }}$ decades of life at the time of diagnosis with female predilection, however ossifying fibroma may affect children and adolescent as well as older adults. ${ }^{1,2}$ It predominantly affects the craniofacial bones, mandible being the most common site of involvement. Reporting here is a case of ossifying fibroma in a female aged 66years with minimal symptoms involving the left maxilla diagnosed with radiograhic and histopathological examination.

\section{Case report}

A female aged 66years reported to the department of Oral Medicine and Radiology of SGT Dental College with the complaint of painless swelling in the upper jaw since 2years that was slowly progressive in nature. The patient had a history of hypertension and was on medication since 4years for the same. Her dental history included extraction of 25, 26, 27 due to caries, around 10years back. Clinical Examination revealed (extra orally), facial asymmetry towards the left side associated with fullness in the middle third of the face, measuring approximately size of $18 \mathrm{~mm} \times 18 \mathrm{~mm}$.Overlying skin was normal and pinchable.

Intraorally, a diffuse expansile lesion involving the left maxillary posterior region, measuring approximately $40 \mathrm{mmx} 30 \mathrm{~mm}$ was extending from the distal aspect of 24 to the tuberosity region with partial obliteration of the buccal vestibule (Figure 1) and normal overlying mucosa. The swelling was non tender and bony hard in consistency. There was partial paraesthesia. 16,17,25,26,27,34,36 and 37 were missing, 11 and 21 were non vital along with generalized attrition. Based on the history and clinical examination, a provisional diagnosis of a benign lesion affecting the left maxilla was arrived at.

The investigations advised included the complete blood picture, intraoral periapical radiograph, maxillary occlusal view, panoramic radiograph and $\mathrm{CBCT}$. Periapical radiograph and maxillary left lateral topographical occlusal view a revealed diffuse patchy radiolucency in region of 26, 27 along with multiple flecks of fine calcifications and hypercementosis was seen with respect to 28 (Figure 2) (Figure 3). Panoramic view revealed radiopacity extending from 24 to tuberosity and encroaching onto the maxillary sinus (Figure 4). To make out the exact extent of the lesion CBCT imaging was planned. CBCT sections showed mixed radiopaque radiolucent (predominately radiopaque) lesion, with well defined borders, extending from 24 till tuberosity region (Figure 5) measuring anteroposteriorly $4 \mathrm{~cm}$,superoinferiorly $5 \mathrm{~cm}$, and mesiodistally around $2.5 \mathrm{~cm}$ in dimension. The pterygoid plates though intact, the lesion however did superimpose on the maxillary sinus. No secondary reaction in the sinus could be appreciated. With a clearer picture on the extent of the lesion, we took the next step towards the management.

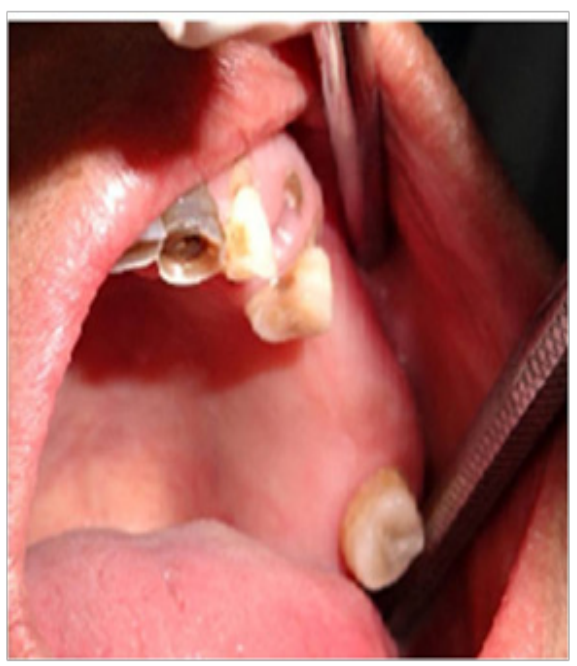

Figure I Partial obliteration of the buccal vestibule.

Incisional biopsy followed by surgical resection of the left posterior maxilla was done under general anaesthesia (Figure 6A-6C). Histopathology revealed proliferating fibroblastic stroma without any mitotic activity and with the deposition of lamellar bone. Connective tissue stroma comprised of plump spindle shaped fibroblasts. Trabecular ossification showed osteoblastic rimming (Figure 7). The result of which confirmed the diagnosis of ossifying fibroma and the patient is under regular follow up. 


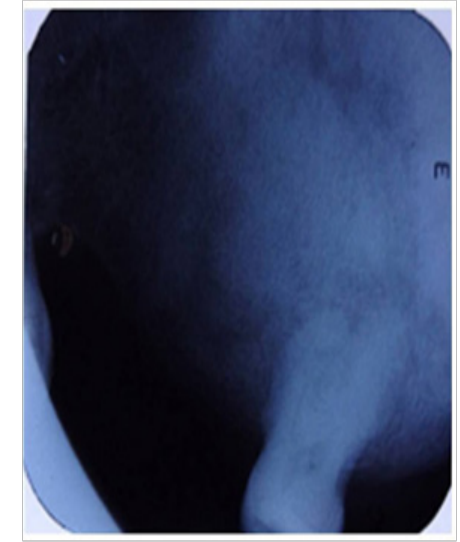

Figure 2 The complete blood picture, intraoral periapical radiograph, maxillary occlusal view.

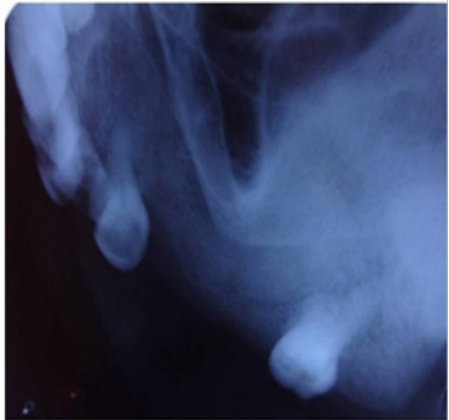

Figure 3 Periapical radiograph and maxillary left lateral topographical occlusal view.

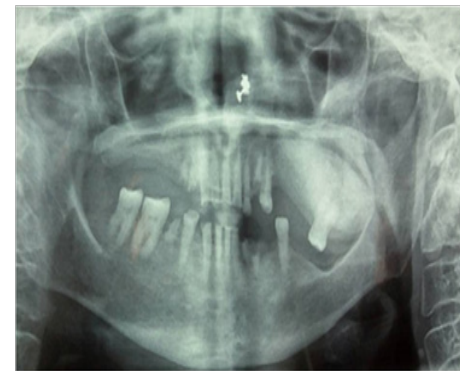

Figure 4 Panoramic view revealed radiopacity extending from 24 to tuberosity and encroaching onto the maxillary sinus.

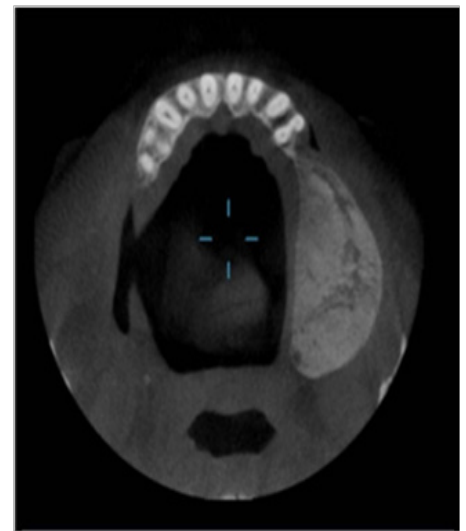

Figure 5 CBCT sections showed mixed radiopaque radiolucent (predominately radiopaque) lesion, with well defined borders.

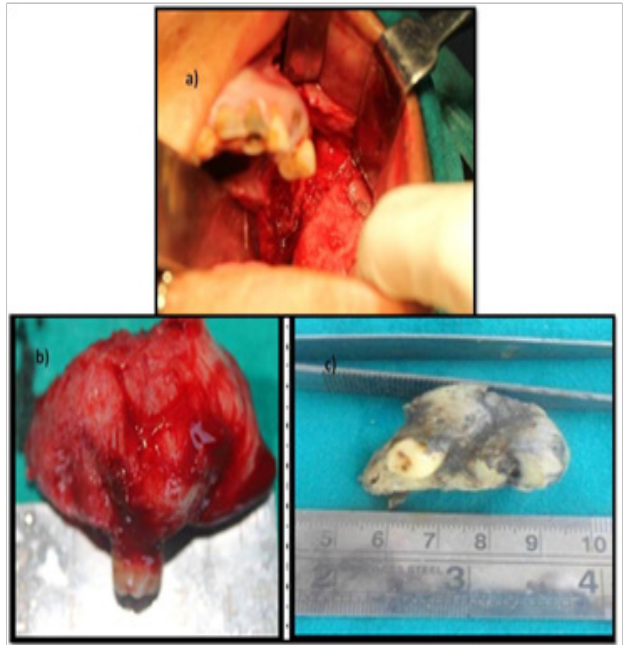

Figure 6 Left posterior maxilla was done under general anaesthesia.

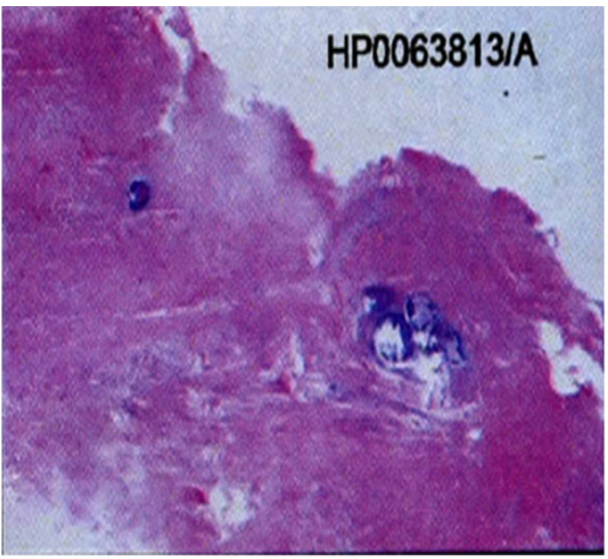

Figure 7 Trabecular ossification showed osteoblastic rimming.

\section{Discussion}

Sciubba, Yih ${ }^{3,4}$ attributed the first description of OF to Menzel in 1872 as a rare benign primary bone tumor that affects most commonly the jaws. However the term, OF was first used by Montgomery ${ }^{5}$ in 1927 , by which the lesion is currently known.

In 1971, the World Health Organization (WHO) classified four types of cementum containing lesions: fibrous dysplasia, ossifying fibroma, cementifying fibroma and cemento-ossifying fibroma. ${ }^{6}$ According to the second WHO classification, benign fibro-osseous lesions in the oral and maxillofacial regions were divided into two categories, osteogenic neoplasms and non-neoplastic bone lesions; cementifying ossifying fibroma belonged to the former category. However, the term "cementifying ossifying fibroma" was reduced to ossifying fibroma in the new WHO classification in 2005. 7,8

Ossifying fibroma develops from the pleuripotent mesenchymal cells that are capable of forming cementum, bone and fibrous tissue. ${ }^{1,9-11}$ According to Ono et al., ${ }^{12}$ the histogenesis of OF consists of two possible origins, the excessive proliferation of the cells of periodontal ligament and a metaplastic process occurring in the connective tissue fibres (non periodontal in origin), the former being more common. OF affects the jaws, could be related to an extensive mesenchymal cellular induction in to bone and cementum, required 
in odontogenesis. Hence an error in the tissue induction process, may lead to the development of $\mathrm{OF}$ in the jaws. ${ }^{1}$ Although the precise triggers are still unknown, other suggested factors include trauma, previous extractions and prior existence of periodontitis. OF has female predilection and occurs in patients of wide age range (10$59 \mathrm{yrs}$, mean 32yrs) with peak incidence in the third or fourth decades of life. Most common site of occurrence is the mandibular premolarmolar region, and about $30 \%$ of cases occur in the maxilla.

Our present case is nearly three decades older than the reported peak incidence and involved the maxilla in a female patient. Juvenile aggressive $\mathrm{OF}$ is a subtype of OF, arises in children (early onset), more aggressive clinically, exhibit rapid growth and erosion or invasion of the adjacent tissue and more vascular on histopathologic examination. Ossifying fibromas with size over $80 \mathrm{~mm}$ in their greatest diameter have been termed as "Giant Ossifying Fibroma". ${ }^{13,14}$ Central ossifying fibromas are slow growing and asymptomatic until they cause expansion. One remarkable finding that the maxillary lesions are large at the time of diagnosis could be attributed to the capacity of the tumor to expand freely within the maxillary sinus similar to our case. The patient did not present with significant clinical symptoms, despite the large size of the lesion encroaching the sinus. OF exhibit markedly varying radiographic patterns based on the amount of mineralized tissue and degree of progression and maturation of the lesion at the time of presentation. Radiographically, the lesion is well circumscribed and initially seen as an osteolytic image followed by gradual transformation into a mixed lesion finally in cases of long duration, it becomes radiopaque, the final stage being considered as exceptional. ${ }^{11}$ It has been stated that the typical OF grows in a centrifugal fashion, producing a ball like circular lesion, enlarges in all directions thereby causing the cortical expansion parallel to the tumor mass. The latter clinical finding can be used to differentiate OF from fibrous dysplasia which causes a linear expansion of the cortex, in which the expanded cortex cannot maintain the exact parallel relationship to the tumor mass.

\section{Eversole et al. ${ }^{15}$ reported 6 variations in the radiographic patterns of OF}

a. The most prevalent pattern has a unilocular radiolucency containing radiopaque foci with neither root divergence nor root resorption. The radiopaque foci yield a target appearance with dense foci of ground glass opacification and irregular opacification.

b. The second common pattern shows a well delineated unilocular radiolucency without radiopaque foci superimposed over the intact roots without causing the root divergence or root resorption.

c. A radiolucency with central opacifications, root divergence and/ or root resorption. The radiopaque foci yield a target appearance with dense foci of ground glass or irregular opacifications.

d. Massive,expansile lesion larger than 5cm,exhibited well-defined margins with ground glass or mottled radiopacities. These lesions were classified as active or aggressive $\mathrm{OF}(\mathrm{AOFs})$.

e. A multilocular radiolucency with/without root resorption.

f. A unilocular radiolucency interposed between divergent or resorbed roots. Waldron CA and Giansanti ${ }^{16}$ stated that all OF invariably show a thin radiolucent line surrounding the lesion representing capsule and sometimes a sclerotic rim present within the host bone at the margin that may be smooth and delicate or it may be slightly irregular, more diffuse and of varying thickness. In our present case the $\mathrm{CBCT}$ showed a mixed radiolucent radiopaque lesion of size $4 \mathrm{~cm} \times 5 \mathrm{~cm} \times 2.5 \mathrm{~cm}$ encroaching the maxillary sinus surrounded by a radiolucent line causing expansion and thinning of the cortical plates, mimicking the Eversole type 4 OF.

Histologically the OF are encapsulated, well circumscribed and basically composed of many delicate collagen fibers with whorled arrangement interspersed by large number of actively proliferating fibroblasts. The connective tissue characteristically presents spherical deposits of calcified material that may increase in number, enlarge and coalesce as the lesion matures accounting for the increasing radiopaqueness of the lesion on the radiograph and also show osteoblastic rimming similar to the present case reported here.

The radiologic differential diagnosis of OF necessarily includes the radiolucent lesions and lesions that contain radiopacities within well defined radiolucent mass depending on the degree of maturation at the time of presentation. Keeping the present case in view, lesions like FD, Chondrosarcoma, Osteosarcoma, Benign cementoblastoma, Pindborg tumor, Gorlin cyst and PCOD may be included under differential diagnosis. The highly cellular nature of the stroma reflects the aggressive nature of these lesions and demands complete surgical excision to obviate local recurrence. This can be achieved by enucleation and curettage of smaller lesions and radical resection for larger lesions. The wide variation in the reported rates of recurrences of $14-38 \%$ could be due to incomplete removal, variations in the follow up period. ${ }^{17-19}$

\section{Conclusion}

The ossifying fibroma of the maxilla is an uncommon, benign fibro osseous tumor, one such case of active OF in a female aged 66years with minimal symptoms is reported. CBCT played an important role in determining the extent of the lesion, diagnosis and proper management.

\section{Acknowledgements}

None.

\section{Conflict of interest}

Author declares that there is no conflict of interest.

\section{References}

1. Marx RE, Stern D. Oral and maxillofacial pathology. A rationale for diagnosis and treatment. Chicago: Quintessence; 2002. p. 789-795.

2. Gurol M, Uckan S, Guler N, et al. Surgical and reconstructive treatment of a large ossifying fibroma of the mandible in a retrognathic patient. Journal of Oral Maxillofacial Surgery. 2001;59(9):1097-1100.

3. Sciubba JJ, Younai F. Ossifying fibroma of the mandible and maxilla: review of 18 cases. J Oral Pathol Med. 1989;18(6):315-321.

4. Yih WY, Pederson GT, Bartley MH. Multiple familial ossifying fibromas: relationship to other osseous lesions of the jaws. Oral Surg Oral Med Oral Pathol. 1989;68(6):754-758.

5. Montgomery AH. Ossifying fibroma of the jaw. Arch Surg. 1927; 15(1):30-44.

6. Pindborg JJ, Kramer IRH. Histological typing of odontogenic tumors, jaw cysts and allied lesions. In: International histological classification of tumors. Geneva: WHO; 1971:31-34. 
7. Kramer IRH, Pindborg JJ, Shear M. Neoplasm and other lesions related to bone. Histologic typing of odontogenic tumors. World Health Organization. New York: Springer-Verlag; 1992:28-31.

8. Reichart PA, Philipsen HP, Scuibba JJ. The new classification of head and neck Tumors (WHO)- any changes? Oral Oncol. 2006;42(8):757-758.

9. Eversole LR, Leider AS, Nelson K. Ossifying fibroma: a clinicopathologic study of sixty-four cases. Oral Surg Oral Med Oral Pathol. 1985;60(5):505-511.

10. Mintz S, Velez I. Central ossifying fibroma: an analysis of 20 cases and review of literature. Quintessence Int. 2007;38(3):221-227.

11. Waldron CA. Fibro-osseous lesions of the jaws. J Oral Maxillofac Surg. 1993;51:828-835.

12. Ono A, Tsukamoto G, Nagatsuka H. An immunohistochemical evaluation of BMP-2,-4, osteopontin,osteocalcin and PCNA between ossifying fibromas of the jaws and peripheral cement-ossifying fibromas on the gingival. Oral Oncology. 2007;43(4):339-344

13. Van Heerden WF, Raubenheimer EJ, Weir RG, et al. Giant ossifying fibroma: A clinicopathologic study of 8 tumours. J Oral Pathol Med. 1989;18(9):506-509.
14. Khanna JN, Anarade NN. Giant ossifying fibroma: Case report on a bimaxillary presentation. Int J Oral Maxillofac Surg 1992;21(4):233-235.

15. Eversole LR, Merrell PW, Strub D. Radiographic characteristics of central ossifying fibroma. Oral Surg Oral Med Oral Pathol. 1985;59(5):522-527.

16. Waldron CA, Giansanti JS. Benign fibro-osseous lesions of the jaw :a clinical-radiologic review of sixty-five cases. Oral Surg Oral Med Oral Pathol. 1973;35(2):340-350.

17. Liu Y, Wang H, Zang Z, et al. Ossifying fibroma of the jaw bone: 20 cases. Dentomaxillofacial Radiology. 2010;39(1):57-63.

18. Triantafillidou K, Ventis G, Karakinaris G, et al. Ossifying fibroma of the jaws: a clinical study of 14 cases and review of literature. Oral Surg Oral Med Oral Pathol Oral Radiol. 2012;114(2):193-199.

19. Gunaseelan R, Anantnarayanan P, Ravindramohan E, et al. Large cement-ossifying fibroma of the maxilla causing proptosis: a case report. Oral Surgery, Oral Pathology, Oral Radiology and Endodontology. 2007;104(4):e21-e25. 